Voix et Images

volxetimages

\title{
UN HOMMAGE « COLLOQUIALEMENT » DISTINGUÉ
}

" Le tombeau de Charles Baudelaire " de Nelligan

A “COLLOQUIALLY” DISTINGUISHED TRIBUTE

NELLIGAN'S "LE TOMBEAU DE CHARLES BAUDELAIRE"

UN HOMENAJE ‘COLOQUIALMENTE’ DISTINGUIDO

"LA TUMBA DE CHARLES BAUDELAIRE”, DE ÉMILE NELLIGAN

\section{PATRICK THÉRIAULT}

Volume 42, numéro 3 (126), printemps-été 2017

URI : https://id.erudit.org/iderudit/1041050ar

DOI : https://doi.org/10.7202/1041050ar

Aller au sommaire du numéro

Éditeur(s)

Université du Québec à Montréal

ISSN

0318-9201 (imprimé)

1705-933X (numérique)

Découvrir la revue

Citer cet article

THÉRIAULT, P. (2017). UN HOMMAGE « COLLOQUIALEMENT » DISTINGUÉ :

« Le tombeau de Charles Baudelaire » de Nelligan. Voix et Images, 42(3),

103-116. https://doi.org/10.7202/1041050ar

\section{Résumé de l'article}

Le projet de sonnet en hommage à l'auteur des Fleurs du mal que Nelligan a laissé sous le titre « Le tombeau de Charles Baudelaire " n'a guère retenu l'attention des commentateurs, qui n'en ont offert aucune lecture soutenue. Son style et son imaginaire insolites témoignent pourtant d'un travail d'élaboration - au sens psychique et artistique du mot — indéniable, même remarquable, qui confère à cette " ébauche " non seulement l'unité de structure nécessaire à son fonctionnement et à son authentification comme poème, mais surtout un rare degré d'originalité. L'auteur démontre qu'à travers l'esthétique étrangement « locale » ou " colloquiale » de ce poème, Nelligan essaie, par un ingénieux et paradoxal tour de main, de se positionner avantageusement dans l'espace symbolique de la modernité baudelairienne. 


\section{UN HOMMAGE "COLLOQUIALEMENT» DISTINGUÉ \\ "Le tombeau de Charles Baudelaire" de Nelligan}

\section{PATRICK THÉRIAULT \\ Université de Toronto}

J'ai grandi dans le goût bizarre du tombeau $[\ldots]^{1}$.

[...] (Car le tombeau toujours comprendra le poète) $[\ldots]^{2}$.

Le tombeau de Charles Baudelaire

Je rêve un tombeau épouvantable et lunaire

Situé par les cieux, sans âme et mouvement,

Où le monde prierait et longtemps luminaire

Glorifierait, mythe ou gnome, sublimement.

Se trouve-t-il bâti colloquialement

Quelque part dans Ilion ou par le planisthère?

Le guenillou dirait un elfe au firmament,

Farfadet assurant le reste, Planétaire!

Ô chantre inespéré des pays du soleil,

Le tombeau glorieux de son vers sans pareil

Soit un excerpt tombal, ô Charles Baudelaire.

Je m'incline en passant devant lui pieusement,

Rêvant, pour l'adorer, un violon polaire

Qui musicât ses vers, et perpétuellement.

Ô cygne inespéré des pays du soleil, Que l'excerpteur glorieux de ton tombeau vermeil

Soit maigre et pâle stèle, ô Charles Baudelaire.

1 Émile Nelligan, Euvres complètes, t. I: Poésies complètes, 1896-1941, édition critique établie par Réjean Robidoux et Paul Wyczynski, Montréal, Fides, coll. «Le vaisseau d'or», 1991, p. 252.

2 Charles Baudelaire, Euvres complètes, texte établi, prêsentê et annoté par Claude Pichois, Paris, Gallimard, coll. «Bibliothèque de la Pléiade», 1975, p. 35. 
Je m'incline en passant devant toi pieusement,

Rêvant pour $t^{\prime}$ adorer un violon lunaire

Qui musicât tes vers et iatoulalant ${ }^{3}$.

Le sonnet ou projet de sonnet en hommage au poète des Fleurs du mal que Nelligan a laissé sous le titre «Le tombeau de Charles Baudelaire» n'a guère retenu l'attention des commentateurs, qui n'en ont offert aucune lecture soutenue. La datation incertaine de sa composition, qu'on hésite à situer avant ou après l'internement de son auteur en août 1899, son état apparent d'inachèvement et plus encore l'opacité de son style et de ses références semblent expliquer ce relatif désintérêt critique. De toute évidence, s'ils ont été reconnus par certains, l'«éclatement visionnaire sans précédent ${ }^{4}$ » et la «surabondance de signification ${ }^{5}$ » dont il est le lieu ont moins encouragé qu'inhibé l'effort herméneutique. On peut le regretter, car, à l'examen, la langue et l'imaginaire insolites du «Tombeau de Charles Baudelaire» témoignent d'un travail d'élaboration - au sens psychique et artistique du mot - indéniable, même remarquable, qui confère à cette «ébauche» non seulement l'unité de structure nécessaire à son fonctionnement et à son authentification comme poème ${ }^{6}$, mais surtout un rare degré d'originalité. S'il est vrai que l'étonnante «couleur locale» dont ce texte est imprégné le détache sans ambiguïté du corpus nelliganien, la vision onirique à motif cosmique qu'il déploie, en se détournant des poncifs du genre funéraire, l'éloigne encore plus nettement, sans doute, de la vaste production des poèmes dédicatoires de la modernité. C'est un fait que, dans l'espace générique et institutionnel où il s'inscrit, celui du tombeau et, plus précisément, du tombeau baudelairien, il représente une proposition esthétique très intéressante, car résolument distinctive: $c^{\prime}$ est sans complexe qu'il peut rivaliser en inspiration, en raffinement et en singularité - bref, en nouveauté, d'après le mot qui résume tout l'esprit et la critériologie de la modernité - avec les meilleurs tombeaux poétiques que le dernier quart du XIX ${ }^{\mathrm{e}}$ siècle a vus proliférer, dans le cadre ou sous l'inspiration des recueils collectifs publiés en hommage à Théophile Gautier, en 1873, puis à Baudelaire, en 18967 . C'est ce que nous nous proposons ici de démontrer.

Or rendre compte de l'originalité d'un texte comme celui-ci ne signifie pas seulement caractériser ses principales composantes pour le situer par rapport à la norme esthétique avec laquelle il négocie, par écarts et rapprochements, son identité

3 Émile Nelligan, Euvres complètes, t. I: Poésies complètes, 1896-1941, nouvelle édition entièrement refondue d'après l'édition critique de 1991, préparée par Réjean Robidoux et Paul Wyczynski, Montréal, Fides, coll. «Biblio-Fides», 2012, p. 175. Les passages en italique des deux derniers tercets signalent des variantes par rapport à la version des deux tercets précédents.

4 Paul Wyczynski, «Nelligan et Baudelaire», Réception de M. Adrien Thério, M. Paul Wyczynski, M. Jean Darbelnet et M. Léon Dion à la Société royale du Canada, Ottawa, Société royale du Canada, 1970, p. 52.

5 Réjean Robidoux, Connaissance de Nelligan, Montréal, Fides, coll. «Le vaisseau d'or», 1992, p. 88.

6 Sur l'unité comme critère dans l'économie herméneutique du poème moderne, voir Michael Riffaterre, Semiotics of Poetry, Bloomington/London, Indiana University Press, coll. "Advances in Semiotics», 1978, p. 2-3.

7 Sur le contexte de publication de ces recueils, voir l'introduction de François Brunet à l'édition critique du Tombeau de Théophile Gautier (Paris, Honoré Champion, coll. «Textes de littérature moderne et contemporaine», 2001, p. 11-45). 
poétique. Cela implique aussi de discerner comment, à travers cette négociation esthétique, le "poète» (c'est-à-dire tout ensemble l'inscripteur, le personnage d'écrivain et la personne réelle Nelligan) essaie d'être reconnu; et comment, incidemment, il en vient à se définir et à être défini par la dynamique d'affirmation et d'assignation symboliques que Dominique Maingueneau a analysée sous le nom de "paratopie ${ }^{8}$ ». Car, là comme ailleurs, le tombeau a ceci de particulier, et d'intéressant sur le plan critique, qu'il est une production poétique condensant et surdéterminant les éléments symboliques inhérents à l'acte littéraire: le poète qui investit ce genre ne vise pas seulement à rendre hommage à un maître disparu; il désire aussi s'inscrire dans sa filiation et, sur le plan horizontal, trouver place dans une certaine communauté discursive. Par là même, le genre du tombeau se recommande à lui comme une occasion toute désignée pour affermir son "auctorialité», c'est-à-dire, en suivant le fil de l'étymologie, pour se prendre en charge et s'augmenter, imaginairement et institutionnellement, au double titre de sujet et $\mathrm{d}^{\prime}$ «individu littéraire ${ }^{9} »$. Même inachevé, un tombeau comme celui de Nelligan permet ainsi de mesurer comment les "choix" esthétiques sont fondamentalement conditionnés par les questions symboliques de reconnaissance et d'appartenance, de distinction et d'affirmation. Nous remarquerons plus précisément, après en avoir offert un bref commentaire, qu'à travers l'esthétique «locale» ou «colloquiale» de ce sonnet - qui est tout entier sollicité par la question du lieu -, Nelligan essaie, par un ingénieux et paradoxal tour de main, de se positionner avantageusement dans l'espace symbolique de la modernité baudelairienne.

\section{UNE VISION COSMIQUE... ET «CANADIENNE »}

En publiant et en ébauchant plusieurs tombeaux ${ }^{10}$, Nelligan témoignait d'un intérêt pour un genre dont il ne pouvait, à l'évidence, ignorer l'actualité institutionnelle. Plus qu'aucun autre de ses tombeaux, ce poème donne à penser qu'il avait lu ceux de

8 Voir Dominique Maingueneau, Le discours littéraire. Paratopie et scène d'énonciation, Paris, Armand Colin, coll. «U. Lettres», 2004, p. 70-116; et Trouver sa place dans le champ littéraire. Paratopie et création, Louvain-la-Neuve, Academia-L'Harmattan, coll. «Au cœur des textes», 2016, p. 25-35.

9 Daniel Oster, L'individu littéraire, Paris, Presses universitaires de France, coll. «Écriture», 1997, 239 p. Sur la notion d'autorité en régime littéraire, voir Emmanuel Bouju (dir.), L'autorité en littérature, Rennes, Presses universitaires de Rennes, coll. «Interférences», 2010, 512 p. ; et Alain Brunn, Le laboratoire moraliste. La Rochefoucauld et l'invention moderne de l'auteur, Paris, Presses universitaires de France, coll. «Les littéraires», 2009, en particulier p. 5-31. Dans ce contexte, on ne s'étonnera pas que certains tombeaux poétiques célèbres - par exemple celui que Hugo dédie à Gautier ou bien le Toast funèbre de Mallarmé soient travaillés de l'intérieur par certaines tensions ou questions relatives à l'auctorialité au point d'infléchir leur destination officiellement commémorative ou encomiastique vers une forme d'autoreconnaissance et des luttes de rivalité. Voir la postface de Jean-Marc Hovasse à l'édition critique du Tombeau de Théophile Gautier («Le tombeau de Victor Hugo [Mallarmé lecteur de Victor Hugo]», p. 287-311), ainsi que notre article «Donner de la voix. Vocalité et auctorialité dans Toast funèbre» (Études françaises, vol. LII, nº 3 , 2016, p. 31-51).

10 Voir Paul Wyczynski, Nelligan, 1879-1941. Biographie, Montréal, Fides, coll. "Le vaisseau d'or", 1987, p. 306-314; et Émile J. Talbot, Reading Nelligan, Montréal/Kingston, McGill-Queen's University Press, 2002, p. 53-60. 
Mallarmé et qu'il avait entendu parler du projet, sinon de la réalisation, du Tombeau de Charles Baudelaire. L'idée de ce recueil collectif avait été lancée dès 1892 par les poètes de La Plume, concurremment à la souscription pour l'édification d'une statue à la mémoire de Baudelaire, initiative qui avait suscité, comme on sait, une vive querelle dans la presse française ${ }^{11}$ et qui eut des échos jusque dans le petit monde des lettrés canadiens-français ${ }^{12}$. Du reste, il ne faut pas exagérer l'éloignement culturel du Canada par rapport au méridien, parisien, des lettres: avant même la fondation de l'École littéraire de Montréal en 1895 et malgré l'opacité idéologique des élites politico-religieuses, on trouvait dans la métropole canadienne une foison étonnante de "petites revues» françaises, parmi lesquelles Le Mercure de France, Le Journal, La Revue indépendante, Les Entretiens politiques et littéraires ${ }^{13}$. La ville de Montréal - ou plus exactement l'une de ses improbables banlieues, Sainte-Cunégonde pouvait même se vanter d'être le siège d'une modeste mais dynamique revue, $L^{\prime} E$ Écho des jeunes, qui était assez justement accordée au diapason de l'avant-garde française contemporaine ${ }^{14}$.

Nelligan n'a sans doute pas eu connaissance de toutes ces publications, mais on peut penser qu'il en a feuilleté un bon nombre ${ }^{15}$. En tout cas, empruntant sans grand souci de discernement au romantisme, au Parnasse, au décadentisme et au symbolisme, il manifeste une conception de la modernité qui reflète l'éclectisme de la réception de la production littéraire française au Canada à la fin du XIXe siècle et donc les principales conditions, avec l'école, de son innutrition culturelle. «Le tombeau de Charles Baudelaire», sur ce point aussi, peut servir de témoin. Même si son identité intertextuelle peut être rapportée au modèle baudelairien - qu'il revendique officiellement, et qu'il met spécifiquement en exergue, comme nous le verrons -, il porte la marque évidente de plusieurs influences: par son fantastique noir et hallucinatoire, il est clairement redevable à Poe et à Rollinat; par sa syntaxe elliptique et ses longs adverbes, à Mallarmé; par ses références fantaisistes et sa prédilection pour le mot rare, à Verlaine; de même, par certaines tournures en apparence négligées de son style, il semble se souvenir de Laforgue... La lecture rapprochée de ce texte nous permettra toutefois de constater qu'au-delà de l'effet «centon» ou citationniste qu'il peut ainsi suggérer, il représente une appropriation réussie, car à la fois fidèle et originale, de l'œuvre de Baudelaire en particulier et de la modernité symboliste en général. Incidemment, elle nous permettra aussi de constater que l'innutrition est presque en même temps, chez celui qui a composé l'essentiel de son œuvre en quelques années d'adolescence, métabolisation ou «transmutation profonde ${ }^{16}$ » des influences reçues.

11 Voir André Guyaux (dir.), La querelle de la statue de Baudelaire (août-décembre 1892), Paris, Presses de l'Université Paris-Sorbonne, coll. «Mémoire de la critique», 2007, 712 p.

12 Voir Paul Wyczynski, Émile Nelligan. Sources et originalité de son œeuvre, Ottawa, Éditions de l'Université d'Ottawa, coll. «Visages des lettres canadiennes/Publications du Centre de recherches en littérature canadienne-française de l'Université d'Ottawa», 1960, p. 32-33.

13 Voir Paul Wyczynski, Nelligan, 1879-1941. Biographie, p. 179-182.

14 Voir Michel Pierssens et Roberto Benardi, «L'Écho des jeunes. Une avant-garde inachevée», Études françaises, vol. XXXII, n 3, automne 1996, p. 21-50.

15 Paul Wyczynski, Nelligan, 1879-1941. Biographie, p. 181.

16 Réjean Robidoux, Connaissance de Nelligan, p. 55. 


\section{COMMENTAIRE : PREMIER QUATRAIN}

1. Je rêve un tombeau épouvantable et lunaire

2. Situé par les cieux, sans âme et mouvement,

3. Où le monde prierait et longtemps luminaire

4. Glorifierait, mythe ou gnome, sublimement.

Le premier vers déstabilise d'entrée de jeu les attentes de lecture induites par le thème funéraire du titre: il étonne, par son image cauchemardesque, et détonne, par le heurt de son hiatus et sa prosodie irrégulière (5-7). Mais son aspect énigmatique et inharmonieux s'explique à la lumière du régime poiétique auquel le poète associe d'emblée (et raccorde finalement, au v. 13) son énonciation: le rêve. Employé sans complément prépositionnel, le groupe verbal «rêver» acquiert ici une puissance de suscitation presque performative, et évoque par là une forme de voyance poétique à mi-chemin entre l'hallucination volontaire et l'onirisme traditionnel.

Placée sous le signe, très poesque, de l'épouvante nocturne, la vision que conditionne ce régime de création est fantastique, mais aussi fantaisiste et merveilleuse, en ce qu'elle fait intervenir la figure du gnome. Le tombeau auquel elle renvoie est perçu au loin, dans l'espace, là où l'ensemble de l'univers, "le monde», donne l'impression de rendre hommage pieusement, liturgiquement, à son dédicataire, Baudelaire. À la manière d'un astre «longtemps luminaire», ce monument cosmique irradie (ou "glorifie», au sens métaphorique et selon un emploi inusité, absolu, du verbe) «sublimement ${ }^{17}$ ». Il apparaît aux yeux de l'observateur terrien sous la forme d'un «mythe» ou d'un "gnome», c'est-à-dire d'une réalité alternativement immense ou ténue - ou peut-être indistinctement et équivalemment l'une et l'autre (le mythe, par rapprochement homophonique, pouvant se résorber dans la petitesse de la «mite»...). Travaillée par des effets de perspective déformants, mais conformes en cela à la logique du rêve, cette vision onirique fait ainsi se côtoyer grandeur mythique et ridicule mythologique, comme les «deux infinis» qu'incorpore la symbolique - inspirée par la conception du poète maudit que Nelligan thématise dans le premier tercet - qui voit en Baudelaire une figure de la culmination (artistique) et de la déchéance (sociale).

17 C'est dire qu'au contraire de ce que la syntaxe suggère d'abord (qui place «le monde» en position de sujet de «longtemps luminaire» et de "glorifier»), nous supposons que cette strophe est asyndétique et qu'elle doit être comprise ainsi: "Je rêve un tombeau épouvantable et lunaire/Situé par les cieux, sans âme et mouvement,/Où le monde prierait et [qui] longtemps luminaire/Glorifierait, mythe ou gnome, sublimement.» Notre interprétation se fonde sur le fait que l'isotopie de la luminosité s'associe en priorité au tombeau de Baudelaire, comme il ressort de l'apostrophe du premier tercet et, ici même, de l'association sonore in absentia «luminaire» > «Baudelaire». Sur un plan plus général, il n’est pas interdit de penser que la difficulté ou l'équivocité de l'attribution grammaticale, dans ce quatrain comme dans le suivant, est un effet herméneutique recherché; qu'elle s'inscrit dans les visées poético-conceptuelles d'un auteur qui prétend offrir non seulement une optique, mais une logique ou une rhétorique du fantasme, et qui, comme tel, s'amuse à rendre la fixation des points de vue incertaine, voire indifférente. Sur la multiplicité et la permutabilité des points de vue dans le fantasme, voir Sigmund Freud, "“Un enfant est battu”. Contribution à la connaissance de la genèse des perversions sexuelles", Névrose, psychose et perversion, traduit de l'allemand sous la direction de Jean Laplanche, Paris, Presses universitaires de France, coll. «Bibliothèque de psychanalyse», 1988, p. 219-243. 
Essayons tout de suite d'estimer la valeur, au sens saussurien du mot, de cette vision cosmique, tant il est vrai que, tout en puisant une partie de sa force suggestive dans le hasard du rêve et, peut-être, de la folie naissante, elle est le produit d'un geste d'écriture assez bien coordonné sur le plan esthétique. On notera à ce titre que, si le thème stellaire appartient de plein droit à la rhétorique du poème funéraire - l'étoile, stella, appelant souvent la stèle, stela ${ }^{18}-$, aucun auteur de tombeau baudelairien ne lui donne l'ampleur ni l'importance, vraiment centrales, que lui accorde ici Nelligan. De même, si le thème lunaire n'est pas étranger à l'imaginaire décadent ${ }^{19}$, il n'intervient significativement dans aucune des pièces composant le recueil collectif Le tombeau de Charles Baudelaire. Cela dit, ce qui fait l'intérêt de cette vision cosmique, c'est qu'elle innove tout en rappelant certains éléments thématiques de l'œuvre baudelairienne, par une forme de rappel mimétique qui vaut pour un hommage. Elle n'est pas tout à fait une plongée - ou une contreplongée - dans l'Inconnu... De fait, les passages à motif lunaire ne manquent pas chez Baudelaire. On pense en particulier au vers du deuxième Spleen : " - Je suis un cimetière abhorré de la lune ${ }^{20}$ ». Ce vers, en l'occurrence, est doublement intéressant: en plus de conjuguer le thème lunaire avec l'idée d'horreur, comme c'est le cas chez Nelligan, il résume bien l'incongruité de l'image baudelairienne, qualité ou défaut qui, on le sait, est l'une des marques les plus distinctives de l'esthétique des Fleurs du mal. Or, s'il est une chose que la vision cosmique de Nelligan semble bien exprimer, c'est d'abord cette incongruité. Elle est insolite, étrange, déconcertante - à l'égal, notamment, de cette autre image à motif céleste et funéraire, qu'elle veut peut-être rappeler, qu'on retrouve à la fin $\mathrm{d}^{\prime}$ "Alchimie de la douleur»: "Dans le suaire des nuages//Je découvre un cadavre cher,/Et sur les célestes rivages/Je bâtis de grands sarcophages ${ }^{21}$ ». L'évocation tombale de Nelligan, notons-le au passage pour mieux y revenir plus tard, ne saurait relever de la faute de goût, pas plus que de l'arbitraire: si elle a quelque chose d'aberrant et d'inapproprié, c'est qu'elle semble artistement désignée pour capturer le sens même de cette Beauté baudelairienne à l'aspect monstrueusement «énorme, effrayant, ingénu ${ }^{22}$ !»

18 Pensons par exemple à l'aérolithe en forme de «désastre [dés-astre] obscur» que Mallarmé associe à la stèle d’Edgar Poe. Voir Stéphane Mallarmé, Euvres complètes, t. I: Poésies, édition présentée, établie et annotée par Bertrand Marchal, Paris, Gallimard, coll. «Bibliothèque de la Pléiade», 1998, p. 38.

19 Les principales figures de la modernité poétique (Poe, Baudelaire, Verlaine, etc.) revisitent assez fréquemment, en effet, le thème lunaire, notamment pour exploiter les liens qui l'unissent depuis toujours à la folie. C'est aussi vrai, par ailleurs dans son œuvre, de Nelligan. Sur les rapports entre lune et poésie, qui mériteraient d'être plus amplement étudiés, voir Jean-Pierre Bertrand et Gérard Purnelle, «Poésie sélénique», Martine Jaminon, Jean Richelle et Jean-Marcel Thomas (dir.), Éclats de lune. Entre science et imaginaire, Liège, Maison de la Science ULG, 2013, p. 112-115. Notons que l'imaginaire du «Tombeau de Charles Baudelaire» n'est pas sans rappeler l'univers fantasque du Pierrot lunaire d'Albert Giraud (Paris, Alphonse Lemerre éditeur, 1884, 107 p.), qui inspirera une trentaine d'années plus tard la célèbre composition de Schönberg.

20 Charles Baudelaire, Euvres complètes, p. 73.

21 Ibid., p. 77

22 Ibid., p. 25. Cela, en soi, suffit à dissiper l'impression trompeuse suivant laquelle, dans le cadre de ce qui serait un hommage de pure façade, Nelligan réduirait Baudelaire à un «prétexte» et ne paraîtrait «soucieux que de son propre état d'âme», comme le suppose Réjean Robidoux (Connaissance de Nelligan, p. 58). 


\title{
COMMENTAIRE : SECOND QUATRAIN
}

5. Se trouve-t-il bâti colloquialement

6. Quelque part dans Ilion ou par le planisthère?

7. Le guenillou dirait un elfe au firmament,

8. Farfadet assurant le reste, Planétaire!

Prenant un peu de recul par rapport à sa vision, le poète se demande dans le second quatrain si le tombeau existe sur terre, «colloquialement». La terre ici évoquée (et engrammée phonétiquement dans la rime calembour «planisthère»/ "Planétaire») a une réalité toute relative puisque le nom «Ilion», doublon de la mythique Troie, ne peut pas ne pas faire penser, à travers la référence à Homère, à la littérature. Ce mot revêt donc un sens métapoétique et invite à ce titre à interpréter le poème de façon réflexive. Il en est de même de "planisthère»: tout en rappelant «planisphère», ce néologisme conjoint deux sèmes par trop contradictoires (celui de planéité et celui de volume) pour ne pas aiguiller, à son tour, la lecture vers des questions d'invention et d'énonciation littéraires. De fait, on le verra, ces noms s'inscrivent dans une série convergente de transgressions lexicales à travers lesquelles Nelligan laisse entrapercevoir la trame symbolique de son sonnet.

Particulièrement elliptiques, imprégnés de merveilleux, les deux derniers vers du quatrain défient l'interprétation. On peut néanmoins supposer qu'ils traduisent une impression: celle d'un personnage - à la fois très canadien-français par son appellation de "guenillou ${ }^{23}$ » et très baudelairien par son état de chiffonnier - qui regarde le monument à l'effigie de Baudelaire depuis son point de vue terrestre et croit voir un "elfe» ou un «farfadet» (les deux mots sont vraisemblablement en apposition); ainsi perçu, le poète statufié semble «assur[er] le reste, Planétaire!», c'est-à-dire assumer une tâche («tutélaire», comme c'est le cas chez Mallarmé ${ }^{24}$ ) consistant à présider à une forme d'harmonie cosmique, à veiller sur l'ensemble de l'univers - à l'instar d'un farfadet, une créature réputée gardienne des lieux.

\section{COMMENTAIRE : PREMIER ET SECOND TERCETS}

\author{
9. Ô cygne inespéré des pays du soleil, \\ 10. Que l'excerpteur glorieux de ton tombeau vermeil \\ 11. Soit maigre et pâle stèle, ô Charles Baudelaire. \\ 12. Je m'incline en passant devant toi pieusement, \\ 13. Rêvant pour t'adorer un violon lunaire \\ 14. Qui musicât tes vers et iatoulalant.
}

23 Les dictionnaires hexagonaux du $\mathrm{XIX}^{\mathrm{e}}$ siècle ne recensent pas le terme "guenillou ». Littré réserve seulement une entrée à "guenilloux» (avec un «x»), variante régionale de «baudet»; voir Émile Littré, Dictionnaire de la langue française, en ligne: http://www.littre.org/definition/guenilloux (page consultée le 5 juin 2017).

24 Voir Stéphane Mallarmé, Euvres complètes, t. I: Poésies, p. 38-39. 
Ces tercets ${ }^{25}$, et plus nettement encore leur version primitive, gardent la trace du premier poème-hommage, à motif «solaire», que Nelligan a consacré à l'auteur des Fleurs du mal et qu'il a publié en septembre 1896 sous le titre "Charles Baudelaire $^{26}{ }^{\prime}$. Pour être brusque, le passage qu'ils marquent d'un Baudelaire-lunaire à un Baudelaire-solaire n'est pas incohérent: en plus de s'accorder, sur le plan formel, à la logique interne du sonnet, il est justifié par une isotopie plus générale, qui fait de Baudelaire un «luminaire», c'est-à-dire, littéralement, un astre luminescent. Tout le premier tercet concourt à donner plus de prégnance à l'image - par certains côtés, en effet, solaire et même icarienne ${ }^{27}$ - du poète commémoré: l'apostrophe fait explicitement intervenir le cygne et pointe, par métonymie, à travers le déterminatif aux «pays du soleil», vers la figure de la négresse, autre figure de la marginalité baudelairienne d'ailleurs évoquée dans la célèbre pièce des Tableaux parisiens ${ }^{28}$; en outre, les vers 10 et 11 accusent les traits "maudits» du poète, très clairement à travers la prédication anthropomorphique "maigre et pâle», et plus subtilement à travers l'allusion au vin (auquel renvoie métonymiquement "vermeil») et le calembour final autour du nom du personnage, en l'occurrence iconique, de Vigny («stèle, ô» = «Stello»). Ainsi, tout en faisant prospérer la polysémie que le poème exploite de manière ludique dès son premier vers (par l'anagrammisation partielle du nom du dédicataire: «un tombeau épouvantable et lunaire»), ce tercet s'applique à approfondir la symbolique spirituelle de Baudelaire: s'il en fait un "signe» "inespéré», $c^{\prime}$ est au sens où l'axiologie littéraire (post)romantique pose le génie comme un sauveur, un nouveau Christ descendu du ciel pour assurer la rédemption du peuple tout à la fois élu et déchu de ses descendants en poésie ${ }^{29}$.

Plus que cette symbolique, c'est toutefois, encore là, un mot inédit en français et d'apparence quelque peu barbare qui attire d'abord l'attention: "excerpteur». Dérivant de l'anglais «excerpt» («extrait»), ce néologisme peut être rendu en français par «extracteur». Il recouvre comme tel une dimension métadiscursive et suggère plus précisément une opération herméneutique: la citation comme extraction. Pris en ce sens, l' « excerpteur glorieux» ne correspond pas seulement à un rayon de lumière émanant du tombeau astral de Baudelaire; c'est aussi le poème même de Nelligan, ce poème-ci, qui, par sa nature précisément de "stèle» poétique, cherche à extraire quelque chose de l'œuvre idéale de Baudelaire de sorte qu'elle soit préservée de la mort et de l'oubli. L'épigone formule ainsi le souhait de prolonger l'œuvre de

25 Nous préférons concentrer notre commentaire sur ces deux tercets (seulement), en tendant à les considérer comme plus achevés que les deux autres tercets que nous avons reproduits dans la présentation liminaire du poème. Sur les questions que posent la datation, la transmission et l'établissement du «Tombeau de Charles Baudelaire», voir Émile Nelligan, Euvres complètes, t. II: Poèmes et textes d'asile, 1904-1941, édition critique de Jacques Michon, revue, corrigée et augmentée par André Gervais avec la collaboration de Jacques Michon, Montréal, Bibliothèque québécoise, 2006, p. 453-456.

26 Émile Nelligan, Euvres complètes, t. I: Poésies complètes, 1896-1941, p. 97. Ce sonnet, maladroit dans sa forme et son contenu, présente peu d'intérêt sur les plans critique et esthétique.

27 Voir Charles Baudelaire, «Les plaintes d'un Icare», Euvres complètes, p. 143.

28 Voir Charles Baudelaire, «Le cygne», Euvres complètes, p. 85-87.

29 Par ailleurs, chez Nelligan, la symbolique christique est également très prégnante. Voir Jacques Michon, Émile Nelligan. Les racines du rêve, Montréal/Sherbrooke, Presses de l'Université de Montréal/Éditions de l’Université de Sherbrooke, coll. «Lignes québécoises», 1983, p. 43. 
son maître, quitte à le faire de manière imparfaite, par le truchement de ce qu'il décrit modestement comme une «maigre et pâle stèle».

Le dernier tercet exprime quant à lui la piété du disciple à l'endroit du maître. L'image du "violon lunaire» (ou "polaire», selon l'autre version) déjoue ce que l'expression de ce sentiment aurait pu d'emblée présenter de convenu. Elle est particulièrement riche de connotations en l'occurrence, le violon étant non seulement un motif récurrent de l'imaginaire fantastique des légendes, mais l'instrument magique le plus souvent évoqué dans le folklore canadien-français ${ }^{30}$. Néologisme à caractère onomatopéique, le «iatoulalant» qui clôt le poème, et le prolonge par hyperbate, suggère le son diaboliquement joyeux de ce violon. Coup d'archet ou corde pincée, arco ou pizzicato, le sens de cette harmonie imitative est difficile à nuancer, mais il confère en tout cas, et c'est l'essentiel, une dimension très nettement sonore à la couleur locale que Nelligan imprime à l'ensemble de son tombeau ${ }^{31}$.

\section{SE DISTINGUER «COLLOQUIALEMENT»}

Aussi sommaire soit-elle, notre paraphrase du «Tombeau de Charles Baudelaire» permet d'éclairer les principaux traits de langue et d'imaginaire conférant à sa trame une étrangeté caractérisée. Ces incongruités se désignent à notre attention de lecteur en heurtant nos attentes et constituent comme telles, pour emprunter au vocabulaire de l'herméneutique structurale de Michael Riffaterre, autant d' «agrammaticalités» à travers lesquelles le texte laisse béer sa «matrice ${ }^{32} »$. On aurait tort de les réduire à des «hasards langagiers», même «extraordinaires ${ }^{33}$ ». Du reste, leur statut herméneutique prend d'autant plus de relief qu'elles sont non seulement très saillantes, mais consonantes entre elles: procédant d'un type similaire de transgression (ou d'invention) linguistique, elles donnent en effet l'impression de communiquer entre elles, de faire réseau et de constituer ensemble, à l'échelle du poème, une forme d'armature symbolique. Ce réseau d'agrammaticalités est constitué par la série de cinq lexèmes qui, parce qu'ils renvoient à des mots non attestés dans les dictionnaires de français hexagonaux, peuvent être considérés plus généralement comme des néologismes, à savoir : l'adverbe «colloquialement» (v. 5); les noms «guenillou » (v. 6), «planisthère»

30 Voir Amy McCallum, Le violon enchanté dans les contes littéraires québécois du XIXe siècle, mémoire de maîtrise, Montréal, Université McGill, 2006, f. 4, en ligne: http://digitool.library.mcgill.ca/webclient/ StreamGate?folder_id=0\&dvs=1497552743396 532 (page consultée le 5 juin 2017).

31 On remarquera qu'en plus de s'accorder harmonieusement avec le folklore national, le motif du violon entre en résonance avec l'imaginaire «nerveux» fin de siècle. C'est ainsi, par exemple, qu'il intervient dans la prose décadente d'un Albert Giraud: «Et la danse de Saint-Guy qu'il [le personnage principal, Jean Heurtaut] faisait danser aux mots exigeait du dirigeant violoneux un coup de fébrile archet, un tourmenteur et saccadant rythme, une aiguë névrose jouant sur le dernier fin nerf.» Albert Giraud, Le scribe, Bruxelles, Lucien Hochsteyn éditeur, 1883, p. 34-35.

32 Sur les notions d'agrammaticalité et de matrice, voir Michael Riffaterre, Semiotics of Poetry, p. 1-23. Rappelons que la matrice représente la structure propositionnelle commune autour de laquelle les images principales du poème s'articulent et dont le texte offre diverses variations sans pour autant la révéler en tant que telle. Il revient au lecteur de la délinéer en se prêtant à une comparaison plus ou moins soutenue des images du poème. Paul Wyczynski, Nelligan, 1879-1941. Biographie, p. 312. 
(v. 7), «excerpteur» (v. 10); et le participe présent «iatoulalant» (v. 14). Le nombre (appréciable) et la distribution (presque transversale) de ces néologismes témoignent d'un plan de composition et donc d'une stratégie esthétique somme toute bien établis, capitalisant sur l'emploi de mots inconnus ou non reconnus par la norme linguistique.

Parmi ces agrammaticalités, l'une se détache plus spécialement, et comme spacieusement, de la plage du texte: l'adverbe-hémistiche «colloquialement». Tout recommande ce mot à la réflexion du lecteur, et c'est sans exagération qu'on peut y reconnaître, sinon une «clé» d'interprétation, du moins un formidable condensateur de signification. Sa morphologie même semble l'expression redoublée de son sens: marquée en son centre par un hiatus ("colloquialement»), elle connote quelque chose de bancal, d'imparfait, d'inesthétique, en somme quelque chose de colloquial. Mais le caractère surdéterminé de cet adverbe s'attache d'abord au fait qu'il est une impropriété linguistique ${ }^{34}$ : construction néologique dérivée du latin («loquor») et plus immédiatement de l'anglais («colloquially»), il renvoie au parler de tous les jours, au discours ordinaire, familier, à caractère très souvent conversationnel, qui s'oppose comme tel au discours soutenu et littéraire. Son appariement avec l'adverbe «sublimement» dans le cadre de la rime suggère plus précisément que le «colloquial» peut faire office de catégorie esthétique et, à ce titre, s'opposer à l'idée d'élévation et de distanciation pour connoter le local. De fait, de quelque côté qu'on l'appréhende, stylistiquement, rhétoriquement ou phénoménologiquement, le «colloquial» apparaît étroitement lié à l'idée de lieu comme lieu de discours (perçu comme) le plus naturel et le plus proche du locuteur.

$\mathrm{Si}$, prenant acte de ces sèmes métadiscursifs, on considère l'adverbe «colloquialement» au-delà de son contexte sémantique immédiat, en extrapolant son sens et en le projetant sur les autres dimensions signifiantes du texte, comme y invite tout jeu textuel à caractère réflexif, on constate qu'il «fonctionne» à la manière d'un embrayeur, et ce, sur deux plans : esthétique et paratopique.

Il se désigne comme un embrayeur esthétique dans la mesure où, en plus de faire signe vers la forme du colloque, qui est fréquente dans la production symboliste $^{35}$, il résume à lui seul et de manière très évocatrice la poétique du présent sonnet. Il permet d'abord de caractériser le "cachet canadien ${ }^{36}$ ", le sceau national, idiomatique, que Nelligan appose à son poème en valorisant, à l'encontre de ses tendances

34 Parce qu'il est d'emblée perçu comme une anomalie, le néologisme littéraire, comme le note Riffaterre, revêt un statut herméneutique privilégié; mieux qu'aucun autre élément lexical, il donne accès à la matrice du poème: «il est le signifiant le plus motivé qu'on puisse trouver dans le texte». Voir Michael Riffaterre, La production du texte, Paris, Éditions du Seuil, coll. «Poétique», 1979, p. 74.

35 Pensons par exemple au «Colloque sentimental» de Verlaine (Paul Verlaine, Euvres poétiques complètes, édition établie par Yves-Gérard Le Dantec, Paris, Gallimard, coll. «Bibliothèque de la Pléiade», 1948, p. 97) ou bien au «Colloque entre Monos et Una» de Poe (Edgar Allan Poe, Contes, essais, poèmes, traduction de Baudelaire et de Mallarmé complétées de nouvelles traductions de Jean-Marie Maguin et de Claude Richard, édition établie par Claude Richard, Paris, Robert Laffont, coll. «Bouquins», 1989, p. 563-571).

36 L'expression est de Louis Dantin: «Je regrette que Nelligan n'ait pas au moins démarqué la part imitative de son œuvre en donnant un cachet canadien à ses ressouvenirs étrangers, ou, plus généralement, qu'il n'ait pas pris plus près ses sources habituelles d'inspiration.» Voir Émile Nelligan, Émile Nelligan et son œuvre, préface par Louis Dantin, édition critique par Réjean Robidoux, Montréal, Presses de l'Université de Montréal, coll. «Bibliothèque du Nouveau Monde», 1997, p. 81. 
esthétiques les plus fondamentales et les plus résolument cosmopolites, des références comme «le guenillou» et le «violon polaire». Le «colloquial» qualifie ensuite, au-delà de ces éléments thématiques, le style familier du poème: son rythme par endroits dégingandé (comme au premier vers) ou encore ses expressions prosaïques (comme la participiale «Farfadet assurant le reste, Planétaire!» ou bien la proposition «le monde prierait» - dont le sujet prend spontanément, en français québécois, une résonance populaire et un sens collectif, valant pour «les gens»). Enfin, et c'est le plus déterminant, l'adverbe «colloquialement» capture le sens insolite et l'essence problématique de la Beauté mise en jeu par ce tombeau, cette Beauté baudelairienne qui s'exprime, comme nous l'avons noté, à travers des tropes incongrus, hardis, "épouvantables» en regard de la norme poétique. Or il est très intéressant de noter que Jules Laforgue, dans les notes sur Baudelaire qu'il a laissées, associe ce type d'images à des «américanismes ${ }^{37}$ ». Le poète des Complaintes considère, en effet, que la manière dont l'imagiste Baudelaire transgresse la juste mesure, choque le bon goût français, procède à des mariages de thèmes et de termes à la fois nobles et vulgaires, élevés et avilissants, a quelque chose d'«américain». Son appréciation de Baudelaire, de toute évidence, porte la marque de sa vive sensibilité à Poe, ou plutôt: elle rend compte, avec une justesse peut-être inégalée à l'époque, de l'extrême sensibilité de Baudelaire à Poe. En discernant à son tour et en exploitant mimétiquement en manière d'hommage l'esprit de bizarrerie qui préside à l'art baudelairien de l'image, Nelligan témoigne non seulement d'une acuité critique comparable à celle de Laforgue, mais en plus d'un rare sens de l'opportunité symbolique: au rebours de la très grande majorité de ses contemporains, qui se contentèrent de puiser sans grand effort le matériau de leur tombeau dans les stéréotypes décadents de la «baudelairité $^{38}$ » - en célébrant par exemple Baudelaire l'ensorceleur ou l'empoisonneur, ou bien en imitant certains traits stylistiques plus évidents des Fleurs du mal (la synesthésie, l'hyperréalisme, etc.) - , Nelligan met en exergue une composante à la fois plus subtile et plus déterminante de l'œuvre baudelairienne et, qui plus est, propre à entrer en étroite et «colloquiale» correspondance avec sa réalité symbolique de poète américain ou nord-américain.

Par ailleurs, l'adverbe "colloquialement» s'avère aussi jouer un rôle d'embrayeur paratopique: par son sens et sa sonorité faisant écho à l'idée de «local», il est bien fait pour actualiser la réflexion qui s'articule, avec insistance et à travers tout le poème, autour de l'écriture comme lieu d'assignation et de légitimation de l'identité auctoriale. C'est parce que ce lieu n'est jamais définitivement conquis, parce qu'il

37 Voir Nelson Charest, «L'“américanisme” de Baudelaire chez les poètes québécois au tournant du xxe siècle», AmeriQuests, vol. XI, nº 1, 2014, en ligne: http://ejournals.library.vanderbilt.edu/index.php/ameriquests/ article/view/3829 (page consultée le 5 juin 2017); et Daniel Grojnowski, «L'“américanisme” des Fleurs du mal: le Baudelaire de Laforgue», André Guyaux et Bertrand Marchal (dir.), Les Fleurs du mal. Actes du colloque de la Sorbonne des 10 et 11 janvier 2003, Paris, Presses de l'Université Paris-Sorbonne, 2003, p. 81-93.

38 Voir Catherine Coquio, "La "Baudelairité" décadente: un modèle spectral», Romantisme, vol. XXIII, nº 82, 1993, p. 91-107. De manière plus générale, sur les premiers moments de la réception de l'œuvre de Baudelaire et l'histoire de la "baudelairité», voir l'introduction d'André Guyaux à Baudelaire. Un demi-siècle de lectures des Fleurs du mal (1855-1905), Paris, Presses de l'Université Paris-Sorbonne, coll. «Mémoire de la critique», 2007, p. 11-139. 
reste toujours et irrémédiablement indécis, problématique, dépendant de la dynamique inchoative du désir et de l'énonciation, qu'il s'assimile à une "paratopie». On le sait, Dominique Maingueneau théorise cette notion en prenant acte d'un constat universel: toute prise d'énonciation implique un écart d'ordre symbolique, qu'elle produit et cherche en même temps à combler. En littérature, la représentation résulte et témoigne elle-même de ce processus fondamental: les personnages de la marge et de l'entre-deux, les situations existentielles en porte-à-faux, les oscillations géographiques en tous genres redisent sur le plan de l'énoncé fictionnel l'exil symbolique de l'auteur ${ }^{39}$. Ils reflètent la dynamique de la création qui fait de l'auteur un exilé, et à travers laquelle, réciproquement, avec plus ou moins de succès selon son degré d'inventivité et d'intelligence pratique, l'auteur cherche à s'exiler, à se dire comme exilé ou, pour être plus exact, à assumer son état, obligé, d'exilé. Comme le souligne Maingueneau, par-delà les attendus du romantisme et le mythe de la malédiction littéraire, et «quelle que soit la modalité de sa paratopie, [l'auteur] est quelqu'un qui a perdu son lieu et doit par le déploiement de son œuvre en définir un nouveau, délimiter un territoire à travers son errance même ${ }^{40}$ ».

Un personnage, dans «Le tombeau de Charles Baudelaire», donne une vive expression à cette errance paratopique: le guenillou. Nous y avons reconnu une version locale ou colloquiale d'une figure emblématique de la modernité baudelairienne, une sorte de chiffonnier canadien. Avec lui, les modestes «guenilles» de la marginalité sociale se transforment en glorieuses enseignes d'une identification symbolicoesthétique que l'on a toutes les raisons de qualifier de réussie, en ce qu'elle favorise une appropriation du référentiel baudelairien sur un mode incontestablement créatif, puisqu'il ouvre un accès privilégié à l'Universel (à l'ordre Symbolique que Baudelaire instancie, en tant que Maître, en même temps qu'au Cosmopolitisme qu'il revendique par son esthétique de la modernité), mais sous le signe paradoxal du familier. À travers cette identification, comme à travers toute paratopie marquée par le succès, l'auteur en arrive à «se maintenir étranger ${ }^{41} »$. Pour être plus exact, on pourrait dire qu'en échappant aux postures également typifiantes du nationalisme, d'une part, et de l'exotisme, d'autre part, il s'affirme comme un étrange familier au regard de ses compatriotes et comme un étranger familier au regard des Européens. Aussi bien, en dépit de la portée pragmatique et institutionnelle limitée, si ce n'est inexistante, du présent tombeau, une telle affiliation s'avère donc probante, et on ne s'étonnera pas qu'elle soit contractée ou, pour mieux dire (tant il est vrai que les jeux de mots affectant les noms propres, ici comme ailleurs, ne sont jamais anodins ${ }^{42}$ ), consacrée dans l'anagrammisation de «Nelligan» dans «guenillou ${ }^{43} »$.

39 L'exemple de Hugo auteur des Châtiments et exilé (volontaire), que développe Maingueneau (Le discours littéraire, p. 106-109), est à ce titre particulièrement évocateur.

40 Dominique Maingueneau, Trouver sa place dans le champ littéraire, p. 29.

41 Ibid., p. 60.

42 Sur l'importance du (jeu sur le) nom propre chez Nelligan, voir Jacques Michon, Émile Nelligan. Les racines du rêve, p. 71-77; et sur le nom propre comme porteur de sèmes paratopiques (et, comme tel, sujet à microanalyses), voir Dominique Maingueneau, Trouver sa place dans le champ littéraire, p. 44-47.

43 On remarquera que l'identification Nelligan-guenillou est renforcée par le fait que le guenillou se présente ici comme un personnage non seulement de la vision, mais, comme le poète, de la parole («Le guenillou dirait un elfe au firmament»): c'est même le seul sujet du poème auquel se rapporte un verbe à caractère 
À côté de cette figure d'identification baudelairienne, il faut en citer une autre, qui vient étoffer la paratopie nelliganienne en la bonifiant de nombreuses virtualités intertextuelles: Andromaque. L'héroïne troyenne est en effet suggérée par les références à «Ilion» et au "cygne», ainsi que par le motif même du tombeau, tous éléments impliqués, comme on sait, dans la célèbre pièce des Tableaux parisiens:

Andromaque, des bras d'un grand époux tombée,

Vil bétail, sous la main du superbe Pyrrhus,

Auprès d'un tombeau vide en extase courbée;

Veuve d'Hector, hélas! et femme d'Hélénus ${ }^{44}$ !

En se demandant si le monument qu'il rêve se trouve "bâti colloquialement/ Quelque part dans Ilion ou par le planisthère», le poète cherche à savoir si, chez lui - sur la Terre aussi bien qu'en terre américaine -, Baudelaire n'a pas trouvé son Andromaque, c'est-à-dire l'exemplaire disciple qui, en s'acquittant du devoir de mémoire et de pérennisation que commandent sa personne et son œuvre, s'imposera comme le digne édificateur et gardien de son «mythe ${ }^{45}$ ». La question est rhétorique, dans tous les sens du terme: l'auteur Nelligan, par divers aspects de son texte et à commencer par l'acte même que constitue ce texte sur le plan symbolique, s'adoubant lui-même comme véritable protecteur et promoteur, dans le Nouveau Monde, de Baudelaire (prétention qui, du strict point de vue de l'histoire littéraire, n'est d'ailleurs pas sans fondement). On remarquera à ce titre que la figure d'Andromaque condense une série de sèmes paratopiques qui sont d'ordre non seulement général, mais singulier; elle traduit, de façon assez spécifique, la situation symbolique de Nelligan en tant que poète exilé dans une "colonie», ou en tant qu'auteur de l'entre-deux-pays et de l'entre-deux-rives, pourrait-on dire - l'Épire et le Canada français, le Simoïs et l'Atlantique entrant en résonance dans ce cadre métaphorique où s'entremêlent, elles-mêmes, la fable et l'histoire. Enfin, s'il n'est pas sidéral, l'état dans lequel Baudelaire fixe son héroïne, dans «Le cygne» («auprès d'un tombeau vide en extase courbée»), connote une forme de sidération qui n'est pas sans faire penser à la scène (imaginaire) d'énonciation du présent poème, telle qu'elle est suggérée par le puissant ethos nelliganien: scène où l'auteur, adoptant la pose et la posture typiquement modernes du poète inspiré, apparaît lui aussi «en extase courb[é]» sur un tombeau (textuel) dont il découvre, par quelque vertu mallarméenne de lucidité, la vacuité (symbolique $\left.{ }^{46}\right) \ldots$

explicitement locutoire. Il est aussi intéressant de souligner que la dimension métatextuelle de ce personnage est suggérée par paronomase: "guenillou» rappelant «guenillon», "petit écrit, écrit de peu de valeur»; voir Ëmile Littré, Dictionnaire de la langue française, en ligne: http://www.littre.org/definition/ guenillon (page consultée le 5 juin 2017).

44 Charles Baudelaire, Euvres complètes, p. 86.

45 On aura observé qu'en donnant beaucoup d'initiative aux mots, Nelligan prend certaines libertés avec le mythe: c'est en Épire, où elle est exilée contre son gré, et non à Ilion qu'Andromaque édifie le tombeau à la mémoire de Hector.

46 Seule, peut-être, une dimension déterminante de la "paratopie singulière» de Nelligan n'est pas reflétée par la figure-symbole d'Andromaque: sa dimension linguistique. Mais elle l'est très clairement par l'adverbe «colloquialement»: en tant qu'anglicisme, en effet, celui-ci suffit à «re-marquer» que l'écriture 
C'est un subjectif, et les spectacles de l'âme l'intéressent beaucoup plus que le cosmos extérieur ${ }^{47}$.

On l'aura constaté: la "surabondance de sens ${ }^{48}$ » que certains commentateurs de Nelligan ont signalée dans "Le tombeau de Charles Baudelaire», sans vraiment s'aventurer à la sonder, est bien réelle. Si elle ne se laisse pas résorber dans quelque signifié premier ou dernier - scintillant sépulcre qu'il reviendrait à l'exégète d'excaver et d'ouvrir -, elle n'échappe pas non plus complètement à l'interprétation en se dissolvant - selon une représentation inverse mais tout aussi fantasmatique dans les tourbillons d'air d'une agitation lettriste parfaitement immotivée ou désespérément inconsciente. La brève analyse que nous avons proposée aura permis de repérer dans le texte de Nelligan des points d'insistance et de récurrence, d'ordre thématique aussi bien que formel, qui permettent d'y reconnaître des motifs structurants et même une logique d'ensemble. C'est ainsi que, par la puissance d'allégorisation dont il est doué et dans le disparate byzantin de son matériau imaginaire, ce texte laisse entrapercevoir une vision non seulement poétique, mais "critique", pourrait-on dire, pour signifier qu' elle condense et configure la plupart des éléments, en particulier symboliques, que nous avons délinéés. Dans cette vision, projeté au loin dans l'espace fantastique d'un cosmos aussi vaste que le cosmopolitisme idéalisé de la modernité, le tombeau de Baudelaire se confond avec l'imposant monument que représente pour Nelligan - aux yeux de sa conscience comme dans l'ordre en partie inconscient de son désir - l'Europe glorieuse, lumineuse, "luminaire», de la République des lettres et, plus précisément, Paris, capitale mondiale de la culture, pôle géographique et institutionnel dont un poète canadien-français, même réputé tout "subjectif», avait quelque raison de se sentir «épouvantablement» éloigné. Sans doute est-ce dans l'optique de cette vision que l'hommage de Nelligan à Baudelaire se révèle le plus «colloquialement» distinct ou distingué; il s'offre alors à l'imagination du lecteur comme une stèle bancale qui, apposée au massif monument métropolitain de la modernité baudelairienne, paraît bien «maigre et pâle», en effet, mais qui, par là même, ajoute à l'ensemble une qualité de fragile et mystérieuse originalité.

chez Nelligan est expérience de l'altérité, qu'elle implique une forme d'«expérience de l'étranger» qui l'inscrit - lui, Canadien français, de père anglophone et, qui plus est, d'ascendance non pas anglaise mais irlandaise - dans un double mouvement de déterritorialisation par rapport à son contexte linguistique immédiat et de conquête appropriative des ressources poétiques d'une langue française en elle-même toujours distante, à quelque degré, par le prestige et l'universalité dont elle se rehausse.

47 Louis Dantin, cité dans Émile Nelligan, Émile Nelligan et son œuvre, p. 83; Dantin souligne.

48 Réjean Robidoux, Connaissance de Nelligan, p. 88. 